\title{
Direct transcatheter aortic valve implantation - one-year outcome of a case control study
}

\author{
Janusz Kochman ${ }^{1}$, Łukasz Kołtowski ${ }^{1}$, Zenon Huczek ${ }^{1}$, Piotr Scisło ${ }^{1}$, Leopold Bakoń2, Radosław Wilimski², \\ Bartosz Rymuza ${ }^{1}$, Grzegorz Opolski ${ }^{1}$ \\ ${ }^{1} 1^{\text {st }}$ Department of Cardiology, Medical University of Warsaw, Warsaw, Poland \\ ${ }^{2}$ Department of Cardiac Surgery, Medical University of Warsaw, Warsaw, Poland
}

Postep Kardiol Inter 2014; 10, 4 (38): 250-257

DOI: $10.5114 / p w k i .2014 .46766$

\begin{abstract}
A b s tract
Introduction: Transaortic valve implantation (TAVI) has a well-established position in the treatment of high-risk and inoperable patients with severe aortic stenosis (AS). The TAVI protocol requires a pre-dilatation for native valve preparation.

Aim: To assess the safety and feasibility of TAVI without pre-dilatation and to compare it with the procedure with pre-dilatation.

Material and methods: Out of 101 TAVI patients, in 10 the procedure was performed without balloon predilatation, and 8 patients were included in the analysis. The procedural, echocardiographic, and clinical outcomes were compared with a case control matched cohort (1: 2 ratio). A 12-month follow-up was done in all cases.

Results: The procedure was successfully completed in all patients in the study group (SG), but there was one procedural failure in the control group (CG). All patients received a CoreValve (Medtronic) bioprosthesis. There was a significant immediate decrease in transvalvular gradients (TG) in both study arms after the procedure (SG: mean TG: from $46.0 \pm 14.0 \mathrm{~mm} \mathrm{Hg}$ to $10.0 \pm 4.8 \mathrm{~mm} \mathrm{Hg}$, $p<0.001$; CG: mean TG: from $55.9 \pm 12.0 \mathrm{~mm} \mathrm{Hg}$ to $9.9 \pm 2.9 \mathrm{~mm} \mathrm{Hg}, p<0.001)$. A marked increase in the effective orifice areas was observed in both cohorts (SG: $1.63 \pm 0.13 \mathrm{~cm}^{2}$ and CG: $1.67 \pm 0.25 \mathrm{~cm}^{2}, p=0.75$ ). The periprocedural complication rate was equally distributed in both arms. The 12-month all-cause mortality was $12.5 \%$ in both groups.

Conclusions: The direct TAVI approach seams to be safe and feasible. The clinical and echocardiographic results are not different from those achieved in patients treated with standard TAVI protocol with pre-dilatation.
\end{abstract}

Key words: transaortic valve implantation, pre-dilatation, no pre-dilatation, aortic valve, balloon valvuloplasty.

\section{Introduction}

Over the last couple of years transcatheter aortic valve implantation (TAVI) has emerged as a reasonable alternative to surgical valve replacement (SAVR) in highrisk patients with symptomatic aortic stenosis (AS), and it has proved to be a superior method to conservative treatment in inoperable patients. Despite the increasing number of TAVI procedures performed worldwide, a substantial risk of periprocedural complications is still observed [1]. The currently used TAVI protocol required a balloon pre-dilatation of the native valve before the actual placement of the bioprosthesis. The balloon pre-dilatation itself might cause some complications including ischaemic stroke caused by embolisation of cerebral vessels by liberated particles from the calcified leaflets, conduction disturbances and the need for pacemaker implantation, worsening of left ventricular ejection fraction
(LVEF) after rapid pacing, as well as coronary artery occlusion [2-4]. This approach was questioned by the results of a recently published pilot study that advocated the safety and efficacy of TAVI without balloon pre-dilatation [5]. On the contrary, there are reports indicating that in selected patients TAVI without pre-dilatation was not feasible due to problems with crossing and implantation of the device [6]. Furthermore, a need for balloon post-dilatation due to paravalvular leak was not negligible and occurred in $17-32 \%$ of patients $[5,7]$. This additional step may also carry significant risk of peri-procedural complications, including annular rapture and cerebral embolism, and prolongs the total time of the procedure.

\section{Aim}

So far there are no long-term results of a direct TAVI approach. To add more data to the discussion and to

\section{Corresponding author:}

Łukasz Kołtowski MD, 1 15t Department of Cardiology, Medical University of Warsaw, 1 a Banacha St, 02-097 Warsaw, Poland,

e-mail: lukasz@koltowski.com

Received: 15.05.2014, accepted: 10.06.2014. 
strengthen the scientific evidence behind the simplified TAVI protocol, we conducted an observational study with a 1-year clinical and echocardiographic follow-up of TAVI patients who did not undergo pre-dilatation and compared them with a case-matched cohort with pre-dilatation.

\section{Material and methods}

\section{Patient selection}

From March 2010 to April 2013 there were 101 TAVI procedures performed at the $1^{\text {st }}$ Department of Cardiology, Medical University of Warsaw (Poland). After implantation of the first 70 patients and reaching a plateau in the learning curve a series of 10 consecutive TAVI procedures were performed using 18-F Medtronic CoreValve prosthesis (Medtronic, Minneapolis, Minnesota) without balloon pre-dilatation (study group). This group was matched at a 2 : 1 ratio with conventional TAVI procedures with pre-dilatation (control group). Patient consent was obtained before medical data were processed.

Standard clinical and echocardiographic criteria for TAVI procedure were used including: severe aortic stenosis with average valve area (AVA) less than $1 \mathrm{~cm}^{2}$ or indexed valve area less than $0.6 \mathrm{~cm}^{2} / \mathrm{m}^{2}$ or mean gradient $>40 \mathrm{~mm} \mathrm{Hg}$ or maximum jet velocity $>4.0 \mathrm{~m} / \mathrm{s}$ or velocity ratio $<0.25$ and age above 75 years or surgical risk assessed by logistic EuroScore (European System for Cardiac Operative Risk Evaluation) above 20, echocardiographic of computer tomography imaging confirming aortic annulus diameter ranging from $20 \mathrm{~mm}$ to $29 \mathrm{~mm}$ [8].

\section{Study design}

The clinical data were obtained from medical documentation and included: baseline characteristics, co-morbidities, pharmacotherapy, procedural details, and in-hospital complications.

The follow-up was carried out by the Outpatients Clinic at 30 days, 6 months, and 12 months and included echocardiographic data (paravalvular leak, mean transvalvular gradient, ejection fraction) as well as cardiovascular end-points (incidence of stroke, myocardial infarction, atrioventricular (AV) conduction disturbances, and death). The paravalvular leak (PVL) was assessed using the following echocardiographic grading: 0 - none, 1 trace, 2 - mild, 3 - moderate, 4 - severe.

The study group was compared with a matched control cohort treated at the same centre with a full protocol TAVI that included pre-dilatation. For each case two controls were searched; logistic EuroSCORE, AVA, prosthesis size, and type of vascular access were used as matching factors.

\section{Device and procedure}

All patients included in the study received CoreValve (Medtronic, Minneapolis, Minnesota) porcine leaflet self-expandable bioprosthesis (Figure 1). Details of the device and the technical aspects of the procedure have been published previously [9]. Briefly, TAVI was performed from transfemoral or subclavian access depending on assessment of the calcification, diameter and tortuosity of the vessels in the multislice computed tomography (CT) imaging, and operator preferences. After gaining the vascular access, positioning a temporary pacemaker, and placing a pigtail in the ascending aorta, the valve was crossed with a 0.35 straight guidewire. Thereafter, this wire was exchanged for a support wire with a manually shaped flexible tip positioned in the apex of the left ventricle. In the next stage a bioprosthesis was implanted using fluoroscopy, aortography, and transoesophageal echocardiography (TEE) for accurate positioning. An inability to properly deliver and position of the valve was considered as device failure. The annulus was sized based on multimodality approach using the transoesophageal echocardiography, CT scans, and angiography images. Three CoreValve diameters were used: 26, 29, and $31 \mathrm{~mm}$. In selected cases a post-dilatation was performed to optimise the procedural result, at the
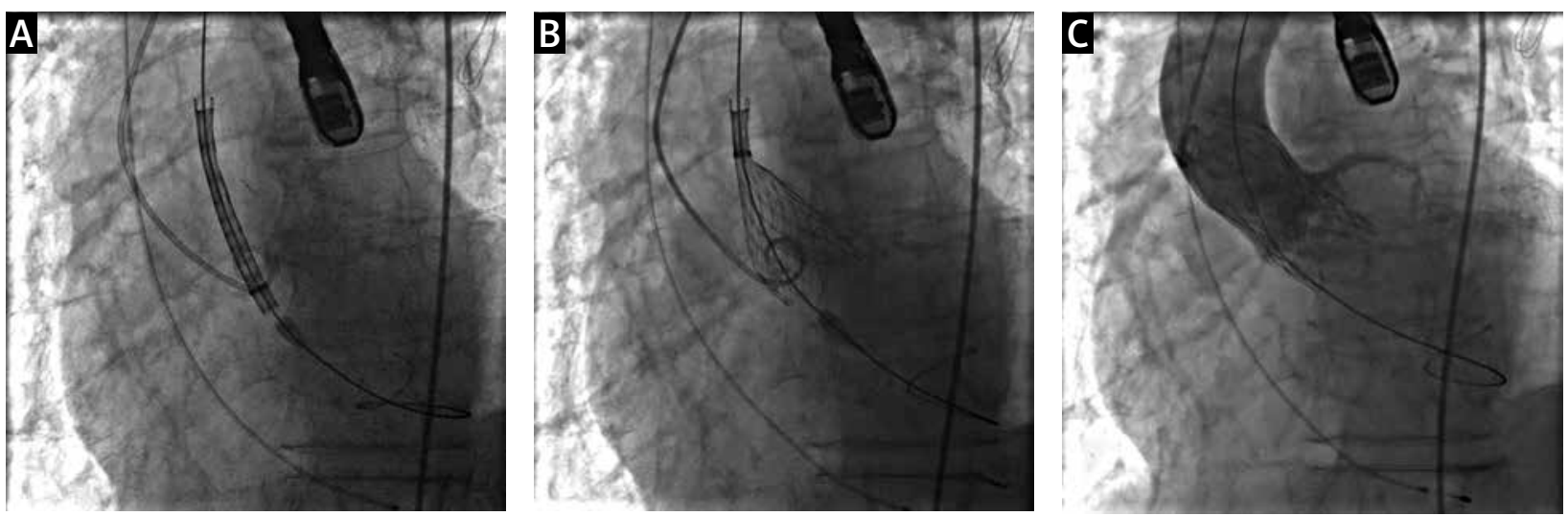

Figure 1. Implantation of bioprosthesis (Medtronic CoreValve, $29 \mathrm{~mm}$ ) in the aortic position. A, B - Left obligue angiographic images with cranial angulation showing final steps of implantation of self-expandabe bioprosthesis. $\mathbf{C}$ - Aortography documenting good valve position and no aortic regurgitation 
discretion of the operator. All procedures were performed under general anaesthesia.

\section{Post-procedural care}

For two to three days after the valve implantation all patients were hospitalised at the Intensive Cardiac Care Unit (ICCU) followed by observation at the General Cardiology Unit. The standard pharmacotherapy comprised acetylsalicylic acid and clopidogrel, the latter was administered for a period of 6 months.

\section{Endpoints and definitions}

The clinical endpoints were defined according to the updated standardized endpoint definitions for TAVI published by the Valve Academic Research Consortium 2 (VARC-2) [10]. Procedural success was defined as the absence of procedural mortality, correct positioning of a single prosthetic heart valve into the proper anatomical location, and intended performance of the prosthetic heart valve (no prosthesis-patient mismatch and mean aortic valve gradient $<20 \mathrm{~mm} \mathrm{Hg}$, and no moderate or severe prosthetic valve regurgitation) [10].

Immediate procedural mortality was defined as an intra-procedural event that resulted in immediate or consequent death $<72 \mathrm{~h}$ post-procedure, procedural mortality consisted of all-cause mortality within 30 days, and long-term mortality was reported after 30 days. Myocardial infarction (MI) was defined as a rise or fall in cardiac troponin with at least one value above the $99^{\text {th }}$ percentile upper reference limit (URL) and with at least one of the following: symptoms of ischaemia, new significant ST-segment-T wave (ST-T) changes or new left bundle branch block (LBBB), an development of pathological $\mathrm{Q}$ waves in the ECG. Peri-procedural myocardial infarction (MI) was limited to $72 \mathrm{~h}$ after procedure, so any $\mathrm{Ml}$ occurring after $72 \mathrm{~h}$ was classified as spontaneous MI. Stroke was defined as a focal or global neurological deficit lasting for more than $24 \mathrm{~h}$ or less than $24 \mathrm{~h}$ if neuroimaging of a new haemorrhage or infarct was available.

\section{Statistical analysis}

Data are presented as mean and standard deviation (SD) or percentages (\%). Differences were assessed by Student's $t$-test or $\chi^{2}$ test, as appropriate, with $\alpha=$ 0.05 as the criterion for significance. For variables with a non-normal distribution, data are presented as median and interquartile ranges and differences were assessed by Wilcoxon signed rank test. Optmatch Package was employed as the statistical case-matching algorithm [11].

\section{Results}

\section{Patient characteristics}

A total of 101 patients were treated with TAVI at the $1^{\text {st }}$ Department of Cardiology of the Medical University of Warsaw between March 2010 and April 2013. In 10 (9.9\%) the procedure was performed without balloon pre-dilatation (study group). Among patients with pre-dilatation (control group) we found corresponding control cases for 8 out of the 10 study group patients. Two patients were excluded due to lack of corresponding match; both received a large valve (diameter of $31 \mathrm{~mm}$ ). Baseline clinical, echocardiographic, and procedural characteristics are presented in Table I. There were no significant differences between the groups. Most procedures were performed from the transfemoral (TF) access (study group: $62.5 \%$ vs. control: $81.3 \%, p=0.36)$. Three valve sizes were used: $26 \mathrm{~mm}, 29 \mathrm{~mm}$, and $31 \mathrm{~mm}$ (Table I).

\section{Periprocedural and in-hospital outcomes}

Procedural success was achieved in all patients in the study group (Table II). There was one failure in the control group, which occurred in an 89-year-old female who was initially hospitalised for decompensated heart failure with AVA $0.3 \mathrm{~cm}^{2}$ and maximal/average pressure gradient of $90 / 60 \mathrm{~mm} \mathrm{Hg}$. The TAVI was complicated by an abdominal aortic dissection secondary to a problem with valve retraction $(29 \mathrm{~mm})$. During releasing phase the valve became dislodged into the ascending aorta and had to be withdrawn for repositioning. Unfortunately, during these manoeuvres the prosthesis became detached from the delivery system. Further attempts to remove the valve were unsuccessful, and finally it was left in the abdominal aorta just above the bifurcation. The control angiography showed an aortic dissection, which was confirmed in the CT angio scan. She was qualified for a stent graft implantation; however, in the next hour she developed a bradycardia followed by a pulseless electrical activity unresponsive to cardiopulmonary resuscitation. Most likely her death was caused by an aortic rapture, but the autopsy study was not performed.

Other severe complications were equally distributed between the two groups and included post-procedural major and life-threatening bleedings (37.5\% of the study group vs. $37.5 \%$ of the control group, $p=1.00$ ) as well as major vascular complications $(12.5 \%$ vs. $12.5 \%$, respectively, $p=1.00$ ).

The mean length of the hospital stay was similar in both study arms (study group vs. control group: $12.3 \pm 4.3$ days vs. $18.1 \pm 15.8$ days, $p=0.32$ ). Details regarding other in-hospital events are provided in Table III.

\section{Echocardiographic outcome}

The post-procedure echocardiographic findings in the study group revealed significant decreases in transvalvular gradients (TG) (mean TG: from $46.0 \pm 14.0 \mathrm{~mm} \mathrm{Hg}$ to $10.0 \pm 4.8 \mathrm{~mm} \mathrm{Hg}, p<0.001$ and peak TG: from 73.4 $\pm 18.6 \mathrm{~mm} \mathrm{Hg}$ to $21.7 \pm 10.2 \mathrm{~mm} \mathrm{Hg}, p<0.001)$. A similar reduction was observed in the control group (mean TG: $55.9 \pm 12.0 \mathrm{~mm} \mathrm{Hg}$ to $9.9 \pm 2.9 \mathrm{~mm} \mathrm{Hg}, p<0.001$ 
Table I. Study population: baseline clinical, echocardiographic and procedural characteristics

\begin{tabular}{|c|c|c|c|}
\hline Parameter & $\begin{array}{c}\text { Study group } \\
\text { (no predilatation) }(n=8)\end{array}$ & $\begin{array}{c}\text { Control group } \\
\text { (predilatation) }(n=16)\end{array}$ & Value of $p$ \\
\hline Age, mean \pm SD [years] & $78.1 \pm 8.4$ & $83.3 \pm 3.7$ & 0.13 \\
\hline Gender (female) (\%) & 50 & 31.3 & 0.41 \\
\hline EuroSCORE, mean \pm SD & $19.7 \pm 5.7$ & $18.6 \pm 7.0$ & 0.72 \\
\hline $\mathrm{BMI}$, mean $\pm \mathrm{SD}\left[\mathrm{kg} / \mathrm{m}^{2}\right]$ & $26.4 \pm 6.1$ & $27.2 \pm 4.6$ & 0.71 \\
\hline \multicolumn{4}{|l|}{ Heart Failure (NYHA class) (\%): } \\
\hline NYHA III & 75 & 43.8 & 0.26 \\
\hline NYHA IV & 0 & 18.8 & 0.26 \\
\hline Coronary artery disease (\%) & 62.5 & 50.0 & 0.68 \\
\hline Chronic kidney disease (\%) & 62.5 & 25.0 & 0.10 \\
\hline Diabetes type 2 (\%) & 50.0 & 37.5 & 0.67 \\
\hline COPD (\%) & 12.5 & 37.5 & 0.35 \\
\hline Atrial fibrillation (\%) & 50.0 & 37.5 & 0.67 \\
\hline Stroke $(\%)$ & 12.5 & 18.8 & 0.59 \\
\hline Peripheral artery disease (\%) & 50.0 & 31.3 & 0.41 \\
\hline Pulmonary artery hypertension (\%) & 12.5 & 6.3 & 0.57 \\
\hline Baseline EF (\%) & 38.4 & 46.8 & 0.08 \\
\hline $\mathrm{AVA}$, mean $\pm \mathrm{SD}\left[\mathrm{cm}^{2}\right]$ & $0.58 \pm 0.15$ & $0.59 \pm 0.17$ & 0.85 \\
\hline Mean AV gradient, mean $\pm \mathrm{SD}[\mathrm{mm} \mathrm{Hg}]$ & $46.0 \pm 14.1$ & $55.9 \pm 12.0$ & 0.09 \\
\hline Peak AV gradient, mean $\pm \mathrm{SD}[\mathrm{mm} \mathrm{Hg}]$ & $84.5 \pm 16.3$ & $73.4 \pm 18.6$ & 0.15 \\
\hline \multicolumn{4}{|l|}{ Valve size, $n(\%)$ : } \\
\hline $26 \mathrm{~mm}$ & $2(25)$ & $4(25)$ & 1.00 \\
\hline $29 \mathrm{~mm}$ & $3(37.5)$ & $6(37.5)$ & 1.00 \\
\hline $31 \mathrm{~mm}$ & $3(37.5)$ & $6(37.5)$ & 1.00 \\
\hline \multicolumn{4}{|l|}{ Access route, $n(\%)$ : } \\
\hline Trans-femoral (TF) & $5(62.5)$ & $13(81.3)$ & 0.36 \\
\hline Trans-subclavian (TS) & $3(37.5)$ & $3(18.7)$ & 0.36 \\
\hline
\end{tabular}

EuroSCORE - European System for Cardiac Operative Risk Evaluation, BMI - body mass index, NYHA - New York Heart Association classification, COPD - chronic obstructive pulmonary disease

Table II. Procedural results

\begin{tabular}{lccc} 
Parameter & $\begin{array}{c}\text { Study group } \\
\text { (no pre-dilatation) }(\boldsymbol{n}=\mathbf{8})\end{array}$ & $\begin{array}{c}\text { Control group } \\
\text { (pre-dilatation) }(\boldsymbol{n}=16)\end{array}$ & Value of $\boldsymbol{p}$ \\
\hline Technical success rate & $8(100)$ & $15(93.75)$ & 0.67 \\
\hline Valve embolization & $0(0)$ & $0(0)$ & 1.00 \\
\hline Conversion to surgery & $0(0)$ & $0(0)$ & 1.00 \\
\hline Post-dilatation & $3(37.5)$ & $2(12.5)$ & 0.55
\end{tabular}

and peak TG: $84.5 \pm 16.3 \mathrm{~mm} \mathrm{Hg}$ to $20.1 \pm 6.4 \mathrm{~mm} \mathrm{Hg}$, $p<0.001)$. These measurements remained stable during the entire follow-up period (Figure 2). The effective orifice area increased to $1.63 \pm 0.13 \mathrm{~cm}^{2}$ in the study group and $1.67 \pm 0.25 \mathrm{~cm}^{2}$ in the control group ( $p=0.75$ ) After 12 months the corresponding values were $1.60 \pm 0.01 \mathrm{~cm}^{2}$ 
Table III. Periprocedural complications and 12-months follow-up

\begin{tabular}{lccc} 
Parameter & $\begin{array}{c}\text { Study group } \\
\text { (no predilatation) }(\boldsymbol{n}=\mathbf{8})\end{array}$ & $\begin{array}{c}\text { Control group } \\
\text { (predilatation) }(\boldsymbol{n}=\mathbf{1 6})\end{array}$ & Value of $\boldsymbol{p}$ \\
\hline Minor bleeding & $5(62.5)$ & $12(75)$ & 0.65 \\
\hline Major bleeding & $2(25)$ & $6(37.5)$ & 0.67 \\
\hline Life threatening bleeding & $1(12.5)$ & $0(0)$ & 0.34 \\
\hline Blood transfusion & $2(25)$ & $6(37.5)$ & 0.67 \\
\hline Minor vascular complication & $1(12.5)$ & $3(18.8)$ & 1.00 \\
\hline Major vascular complication & $1(12.5)$ & $2(12.5)$ & 1.00 \\
\hline Atrioventricular block requiring pacemaker & $2(25)$ & $4(25)$ & 1.00 \\
\hline Myocardial infarction & $0(0)$ & $1(6.3)$ & 1.00 \\
\hline Stroke & $0(0)$ & $0(0)$ & 1.00 \\
\hline In-hospital mortality & $0(0)$ & $1(6.3)$ & 0.67 \\
\hline 12-month mortality & $1(12.5)$ & $2(12.5)$ & 1.00
\end{tabular}
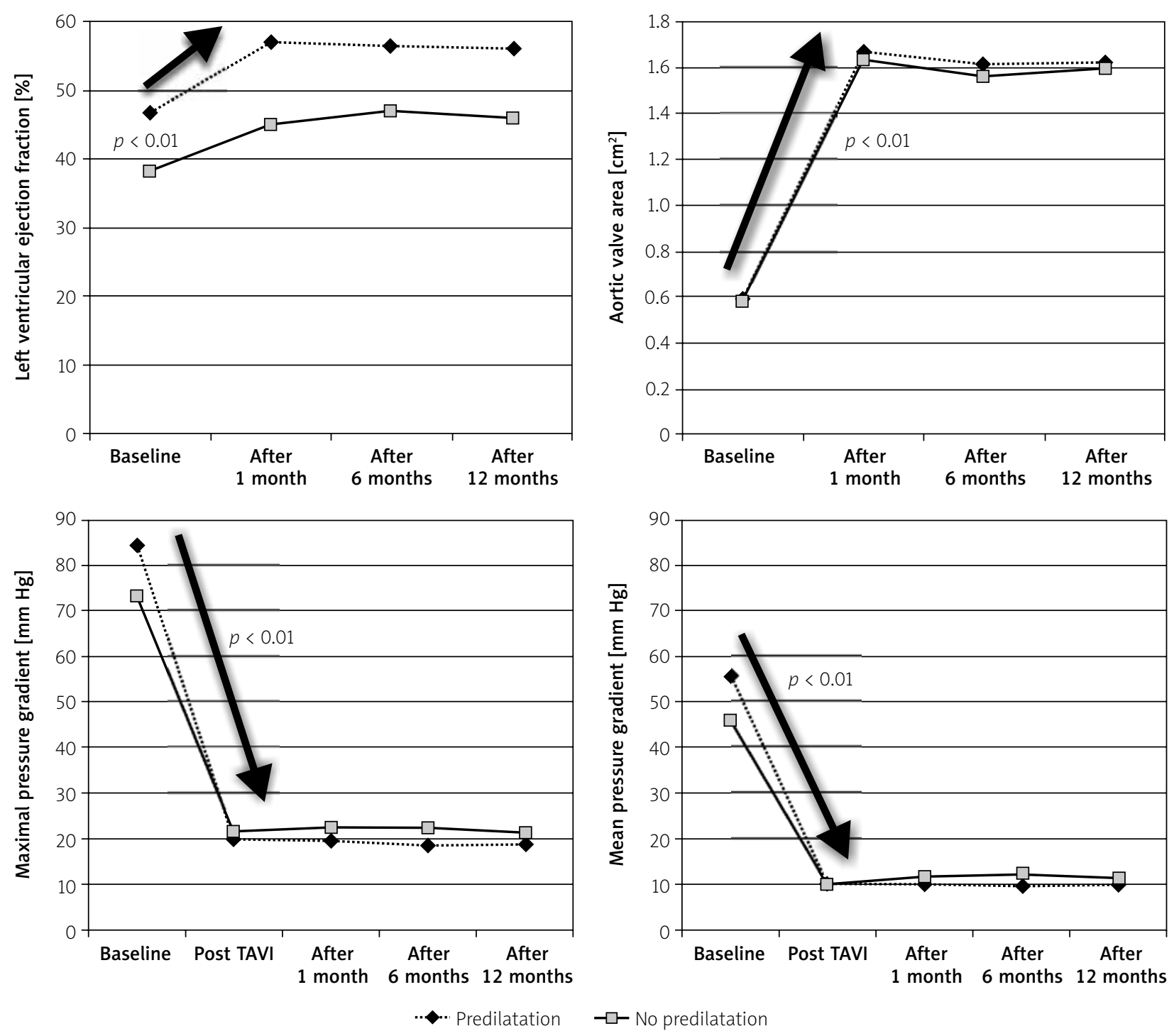

Figure 2. Echocardiographic outcomes at baseline, directly after procedure, at 1 month, at 6 months, and at 12 months. Values directly after the aortic valve implantation were recorded using transesophageal echocardiography, whereas the follow-up measurements were obtained with a transthoracic echocardiography. The arrows indicate changes that are statistically significant $(p<0.01)$ 


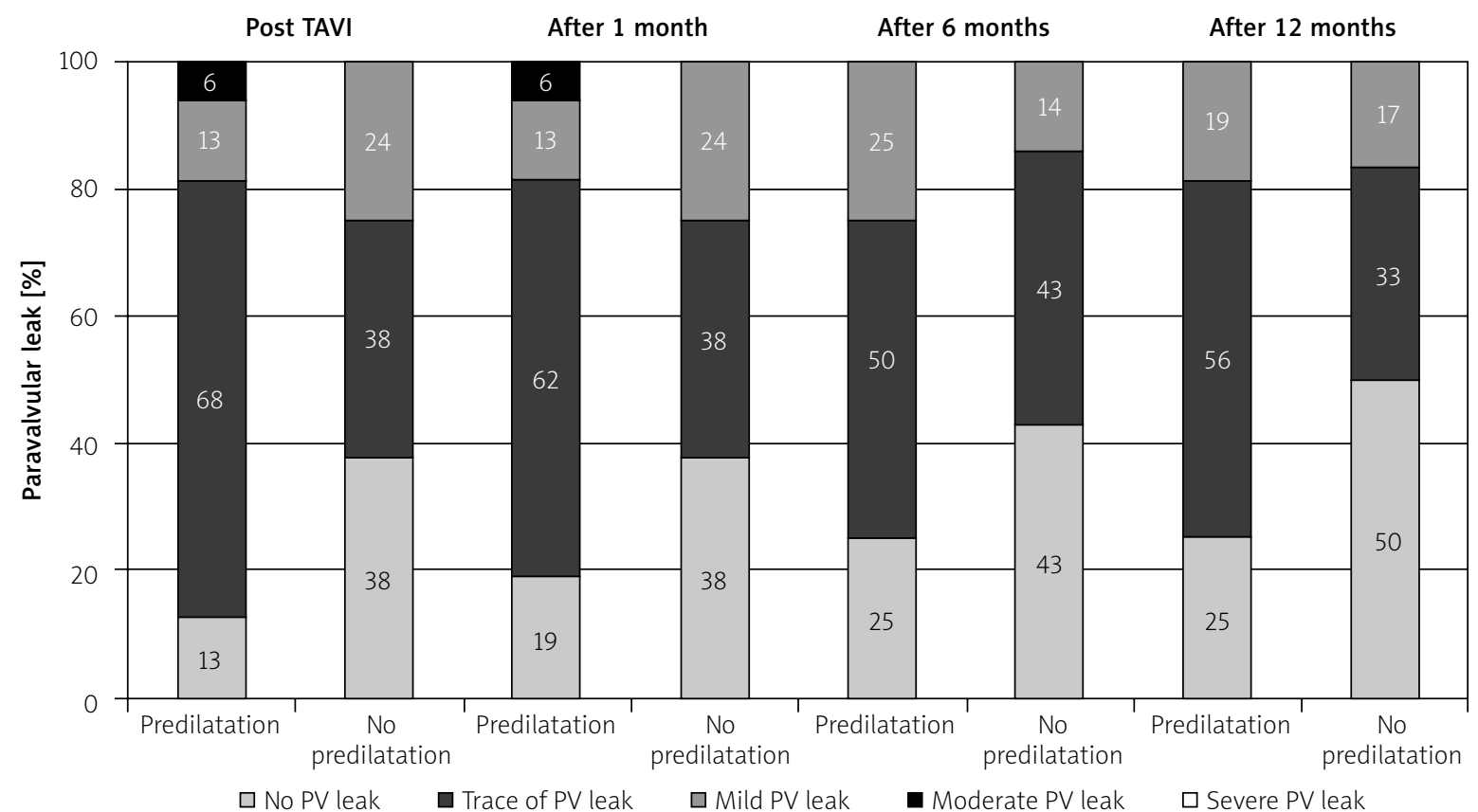

Figure 3. Paravalvular leak directly after procedure, at 1 month, at 6 months, and at 12 months POSt TAVI - after transcatheter aortic valve implantation
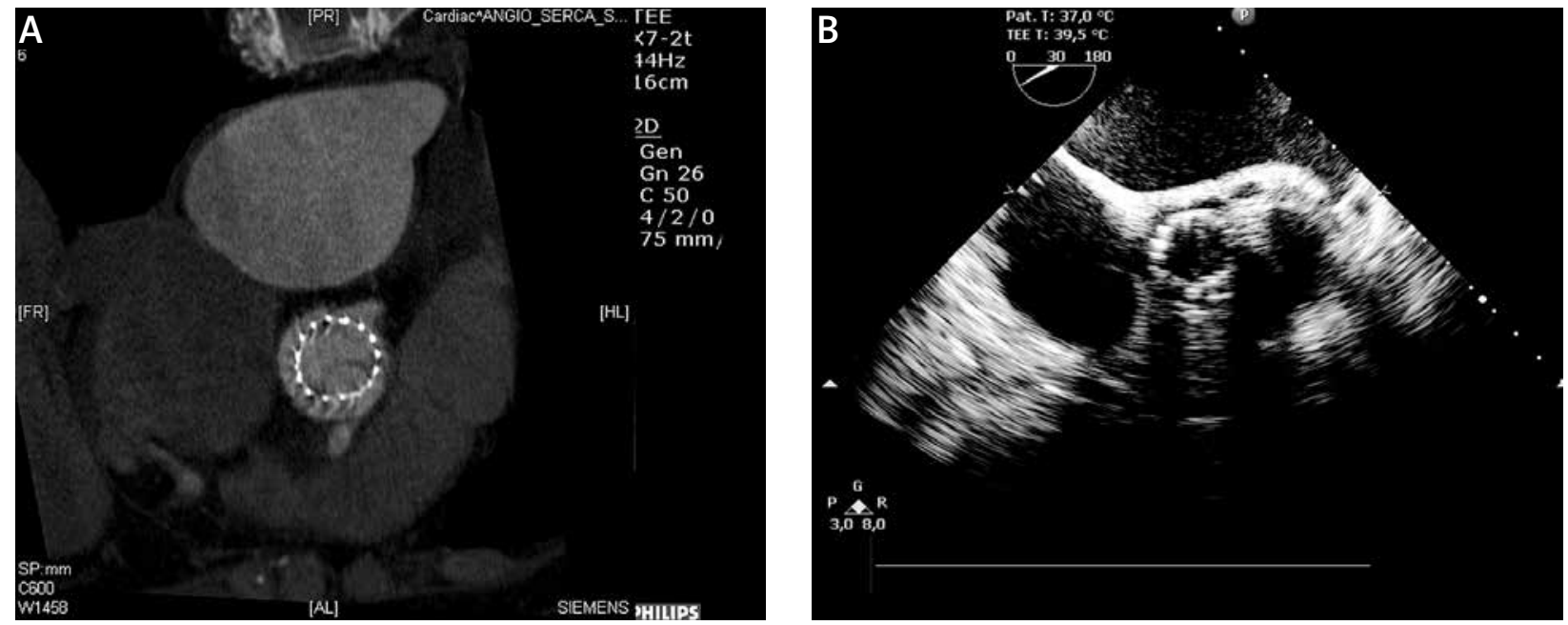

Figure 4. Computed tomography (A) and transoesophageal echocardiography (B) after CoreValve implantation without pre-dilatation. Both imaging modalities show symmetrical bioprosthesis expansion

and $1.62 \pm 0.22 \mathrm{~cm}^{2}$, respectively ( $p=0.82$ ). Similarly, there was a significant improvement in the LVEF between the baseline and 1 month after the procedure in the entire population ( $44 \pm 11.0 \%$ vs. $53 \pm 9.4 \%, p=$ $0.004)$ and it remained unchanged in the follow-up. Mild PVL $(\geq 2+)$ was observed in $25 \%$ of patients in the study group and in $19 \%$ of the control group, directly after valve implantation $(p=1.00)$. After 12 months the corresponding values were $19 \%$ vs. $17 \%$, respectively, $(p=1.00)$. No patient had moderate or severe PVL (Figure 3). An example of a postprocedural TEE and CT are depicted in Figure 4.

\section{0-day and 12-month clinical outcome}

In the 30-day follow-up there was no death in the study group whereas one patient $(6.25 \%)$ died in the control group $(p=1.00)$. The atrioventricular conduction block requiring pacemaker implantation occurred in $25 \%$ of patients in both groups. In the following 12-months (median: 12, 25-75\% interquartile: 9-15) one additional non-cardiac death in the study group (after 9 months) and one in the control group (after 15 months) were observed. Additionally there was one myocardial infarction in the control group. All cause 12-month mortality was $12.5 \%$ in both study arms $(p=1.00)$ (Table III). 


\section{Discussion}

The results of our study show that TAVI without balloon pre-dilatation is safe and feasible and the device success, rate of periprocedural complications, and 1-year survival rate are similar to those seen in patients treated with standard protocol. Equally, the echocardiographic findings confirmed no significant differences with regard to the postprocedural aortic regurgitation, transvalvular gradient, and the effective orifice area, when compared with the control group. Although the study population was small, it has the longest follow-up published so far in the literature.

The routinely used technique for TAVI requires balloon pre-dilatation. This approach is, nevertheless, based purely on expert opinions and was not tested in any randomised, controlled trials. The rationale behind the pre-dilatation comes from the experience with palliative balloon valvuloplasty used in selected cases as a symptom relief therapy or bridge-to-surgery strategy [12, 13]. While the immediate haemodynamic effects confirmed by echocardiographic imaging remain favourable, the procedure itself carries multiple limitations including high restenosis rate, risk of peri-procedural complications, and limited survival benefit $[14,15]$. Pre-dilatation during TAVI was meant to prepare the native valve before introduction of the bioprosthesis by breaking the degenerated leaflets and stretching out the calcified tissue of the aortic wall. Despite this theoretical reasoning, the direct TAVI approach in our study proved to be feasible with high procedural success and immediate/mid-term clinical and echocardiographic outcome. A smooth conduction of the procedure would probably have not been possible if it had not been for the good performance of the CoreValve device, characterised by a relatively low profile and strong radial force, allowing successful deployment and functioning of the valve. This was confirmed by an acute improvement in haemodynamic outcomes, which were equally good when compared with the control group. It is also worth underlining that the rate of PVL did not differ between the patients undergoing TAVI with and without pre-dilatation. Finally, it seems like any potential deformation in the geometry of the bioprosthesis possibly caused by lack of preparation of the native valve did not influence the echocardiographic or the clinical outcomes, at least in the 12-month perspective.

Similar findings were reported by Grube et al. in the paper from a pilot study on the feasibility of TAVI without balloon pre-dilation, which included 60 consecutive patients treated with the Medtronic CoreValve system [5]. The clinical and demographic characteristics of their population were similar to those presented in our study. The technical success rate was slightly better in the no-pre-dilatation group in our population (100\%) compared to that of Grube et al. (81.7\%). However, there were more post-dilations performed in our study $(25.0 \%$ vs. $16.7 \%)$. Grube et al. also reported a $14.3 \%(n=18)$ short-term mortality rate in the predilatation group, which was numerically higher compared with the no predilatation group $6.7 \%(n=4)$. They also revealed higher risk of AV block in the predilatation group: $27.8 \%(n=35)$ vs. $11.7 \%(n=7)$. These findings were not observed in our study, which is probably related to the small sample size of our cohort.

Promising results of direct TAVI approach were also confirmed by Mendiz et al. They reported 51 consecutive high surgical risk patients treated with direct (no predilatation) TAVI (mean age: $79 \pm 8$ years, EuroSCORE: $20 \pm 15 \%$ ( $50 \%$ of patients with EuroSCORE $\geq 20 \%)$ ). The in-hospital and 30-day mortality was low (3.9\%), which is similar to our result. One-fourth of the patients (28\%) required permanent pacemaker implantation, which is again comparable to our cohort (25\%) [7].

Elimination of pre-dilatation seems not only feasible, but it also has a theoretical potential to reduce the peri-procedural risk especially with regard to neurological complications. A number of concerns were expressed regarding the safety of pre-dilatation in highly calcified aorta and valve apparatus. Results of diffusion-weighted neuromagnetic resonance imaging of the brain show a very high prevalence of new cerebral lesions after TAVI [16]. The transcranial Doppler studies performed during the procedure identified high-intensity transient signals (HITS), a surrogate for microembolisation, in up to $100 \%$ of patients [17]. Although most of the embolic events occur during the positioning and implantation phase there are a considerable number of HIST registered during the balloon inflation, which might influence the overall risk of neurological deficit [18].

Although the available results for TAVI without predilatation are promising, we must acknowledge that its definite elimination is rather unlikely. This is mainly because the vast majority of scientific evidence comes from procedures performed using the conventional TAVI protocol. Also, it should not be neglected that there are case reports in which successful procedure was not possible without appropriate ballooning of the native valve [6]. Chan et al. reported two high-risk AS patients who underwent TAVI without balloon pre-dilatation: a 72-yearold female with Takayasu's disease and porcelain aorta, and a 72-year-old male with severe chronic obstructive pulmonary disease. Both attempts were unsuccessful due to difficulties in advancing the delivery system and failure of proper expansion of the valve.

The rather small available body of evidence and still limited experience with direct TAVI makes it very difficult to draw any firm conclusions and to give any recommendation at this stage. Although the results are promising they require further evaluation in larger trials.

There are some important limitations to our study. Firstly, the population size was small. Secondly, the data 
were collected retrospectively and there was lack of randomisation. Nonetheless, the presented results may be considered of some value as they add to the existing body of scientific research helping to understand better the nuances of TAVI procedure.

\section{Conclusions}

Our results indicate that TAVI procedure is safe and feasible when performed without balloon pre-dilatation and provides clinical and haemodynamic improvement comparable to that achieved in TAVI with pre-dilatation.

\section{References}

1. Leon MB, Smith CR, Mack M, et al. Transcatheter aortic-valve implantation for aortic stenosis in patients who cannot undergo surgery. N Engl J Med 2010; 363: 1597-607.

2. Khawaja MZ, Rajani R, Cook A, et al. Permanent pacemaker insertion after CoreValve transcatheter aortic valve implantation: incidence and contributing factors (the UK CoreValve Collaborative). Circulation 2011; 123: 951-60.

3. Vahanian A, Himbert D. Transcatheter aortic valve implantation: could it be done without prior balloon valvuloplasty? JACC Cardiovasc Interv 2011; 4: 758-9.

4. Ben-Dor I, Pichard AD, Satler LF, et al. Complications and outcome of balloon aortic valvuloplasty in high-risk or inoperable patients. JACC Cardiovasc Interv 2010; 3: 1150-6.

5. Grube E, Naber C, Abizaid A, et al. Feasibility of transcatheter aortic valve implantation without balloon pre-dilation: a pilot study. JACC Cardiovasc Interv 2011; 4: 751-7.

6. Chan PH, Mario CD, Moat N. Transcatheter aortic valve implantation without balloon predilatation: not always feasible. Catheter Cardiovasc Interv 2013; 82: 328-32.

7. Mendiz OA, Fraguas H, Lev GA, et al. Transcatheter aortic valve implantation without balloon predilation: a single-center pilot experience. Catheter Cardiovasc Interv 2013; 82: 292-7.

8. Zamorano JL, Badano LP, Bruce C, et al. EAE/ASE recommendations for the use of echocardiography in new transcatheter interventions for valvular heart disease. Eur Heart J 2011; 32: 2189-14

9. Grube E, Laborde JC, Gerckens U, et al. Percutaneous implantation of the CoreValve self-expanding valve prosthesis in highrisk patients with aortic valve disease: the Siegburg first-in-man study. Circulation 2006; 114: 1616-24.

10. Kappetein AP, Head SJ, Genereux P, et al. Updated standardized endpoint definitions for transcatheter aortic valve implantation: the Valve Academic Research Consortium-2 consensus document. J Am Coll Cardiol 2012; 60: 1438-54.

11. Hansen BB, Klopfer SO. Optimal full matching and related designs via network flows. Journal of Computational and Graphical Statistics 2006; 15: 609-27.

12. To AC, Zeng I, Coverdale HA. Balloon aortic valvuloplasty in adults: a 10-year review of Auckland's experience. Heart Lung Circ 2008; 17: 468-74.

13. Jabbour RJ, Dick R, Walton AS. Aortic balloon valvuloplasty: review and case series. Heart Lung Circ 2008; 17 Suppl. 4: S73-81

14. Koning R, Cribier A, Asselin C, et al. Repeat balloon aortic valvuloplasty. Cathet Cardiovasc Diagn 1992; 26: 249-54.

15. Khawaja MZ, Sohal M, Valli $H$, et al. Standalone balloon aortic valvuloplasty: indications and outcomes from the UK in the transcatheter valve era. Catheter Cardiovasc Interv 2013; 81: 366-73.

16. Kahlert P, Knipp SC, Schlamann M, et al. Silent and apparent cerebral ischemia after percutaneous transfemoral aortic valve implantation: a diffusion-weighted magnetic resonance imaging study. Circulation 2010; 121: 870-8.

17. Kahlert P, Al-Rashid F, Dottger P, et al. Cerebral embolization during transcatheter aortic valve implantation: a transcranial Doppler study. Circulation 2012; 126: 1245-55.

18. Erdoes G, Basciani R, Huber C, et al. Transcranial Doppler-detected cerebral embolic load during transcatheter aortic valve implantation. Eur J Cardiothorac Surg 2012; 41: 778-83. 